\title{
Disparities in Genetic Referrals for Breast Cancer among the Asian Immigrant Populations: How Can We Eliminate Them?
}

\author{
Sailaja Kamaraju, MD, $M S^{1^{*}}$, Amanda Jacquart, MS, CGC², Samantha Stachowiak, MS, CGC', Jennifer

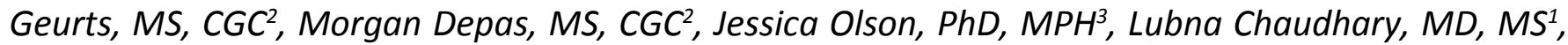 \\ Christopher Chitambar, $M D^{1}$, Joan Neuner, $M D, M P H^{2}$ and Yee Chung Cheng, $M D^{1}$
}

${ }^{1}$ Division of Hematology \& Oncology, Medical College of Wisconsin, Milwaukee, WI, USA

${ }^{2}$ Genomics Sciences \& Precision Medicine Center, Medical College of Wisconsin, Milwaukee, WI, USA

${ }^{3}$ Institute for Health and Equity, Medical College of Wisconsin, Milwaukee, WI, USA

\begin{abstract}
Despite major advances in genetic testing for breast cancer, access to genetic counseling and testing are significantly lower among immigrant and refugee populations. Both patient related barriers and provider-based factors contribute to disparities in genetic referrals and testing. Previous studies addressing genetic referral patterns among the minorities have focused on African Americans, however, there are no reports addressing disparities in genetic referrals and testing in Asian immigrant and refugee populations living in the United States (US). Given the rapid influx of these populations and increasing rates of breast cancer among Asian immigrant populations in the US, this area remains unexplored. This review addresses the current data on familial breast cancer syndromes, describe the various barriers, and attempts to provide suggestions to eliminate the disparities in genetic referral patterns.
\end{abstract}

\section{Introduction}

Genetic counseling practice, referrals to counseling, and genetic testing patterns vary among different populations $[1,2]$. Hereditary cancer risk assessment among immigrant and refugee populations has been impeded by barriers such as low rates of knowledge regarding genetic counseling and limited resources for familial breast cancer risk assessment $[1,2]$. Rates of breast cancer incidence among Asian immigrants and refugees have traditionally been lower than Caucasians, but the gap between populations is narrowing gradually [3]. As breast cancer rates increase among the immigrant populations, continued emphasis is required for the optimal use of screening mammography, identification of high-risk families, and referrals to genetic counseling $[3,4]$. However, this can be somewhat difficult given the low literacy rates, lack of English language proficiency, poor health care access, fear of cancer, and personal pursuit of familial cancer risk assessment in these populations $[3,5,6]$.

Previous studies from the Asian countries (China, Korea, and Japan) utilized vigorous genetic risk assessment and screening efforts, which demonstrated that pathogenic variants are more commonly identified in the BRCA2 gene than in the BRCA1 gene [7]. But in other parts of Asia, e.g., India and Pakistan, despite the availability of genetic testing, the assessment of familial cancer risk is rather under-utilized $[4,5]$. Low rates of breast cancer screening continue even among the residents of Asian immigrant and refugee populations in the US [3]. Furthermore, there are no studies addressing the patterns of familiar cancer syndromes, and genetic assessment among Asian immigrant/refugees despite the rapid increase in the influx of these populations in the US.

Herein, in this article, we attempt to review the current data on the highly penetrant familial breast cancer syndromes described in the Asian immigrant, and refugee populations, and updated referral criteria for genetic counseling and testing. We also describe the barriers, and the future directions to reduce the disparities in genetic referrals for breast cancer among the Asian immigrant populations.

\section{The Data}

\section{Hereditary breast cancer syndromes among Asians}

Hereditary breast and ovarian cancer, BRCA1 and BRCA2:

*Corresponding author: Sailaja Kamaraju, MD, MS, Division of Hematology \& Oncology, Medical College of Wisconsin, Milwaukee, WI, USA, Tel: 414-805-4600

Accepted: May 21, 2019

Published online: May 23, 2019

Citation: Kamaraju S, Jacquart A, Stachowiak S, Geurts J, Depas $M$, et al. (2019) Disparities in Genetic Referrals for Breast Cancer among the Asian Immigrant Populations: How Can We Eliminate Them?. Ann Public Health Reports 3(1):35-40 
Citation: Kamaraju S, Jacquart A, Stachowiak S, Geurts J, Depas M, et al. (2019) Disparities in Genetic Referrals for Breast Cancer among the Asian Immigrant Populations: How Can We Eliminate Them?. Ann Public Health Reports 3(1):35-40

The majority of all breast cancers, in general, are sporadic, with only $5-10 \%$ being inherited. Approximately half of women with hereditary breast cancer, defined as the inheritance of a pathogenic variant in a single cancer susceptibility gene, have pathogenic variants in the BRCA1 and BRCA2 genes, also known as hereditary breast and ovarian cancer syndrome (HBOC). The remaining proportion of hereditary breast cancer is caused by moderate-risk genes, low-penetrant genes, yet undiscovered genes, or rare syndromes [8]. Individuals with a $B R C A 1$ or $B R C A 2$ pathogenic variant, are estimated to have an approximate $43-87 \%$ risk of breast cancer, $15-63 \%$ risk for ovarian cancer, $16-20 \%$ for prostate cancer, $1-7 \%$ risk for male breast cancer, and in some families a $7 \%$ risk or higher for pancreatic cancer $[9,10]$.

Multiple studies have reported the prevalence of $B R C A 1$ and $B R C A 2$ pathogenic variants in Asians. In an unselected cohort of 826 Chinese women with ovarian cancer, there was a reported prevalence of $20.8 \%$ for BRCA1 and $7.6 \%$ for $B R C A 2$ in the women who underwent germline analysis [11]. In carriers of pathogenic variants, late stages of disease (III-IV) was diagnosed in $85.5 \%$ of the patients at a younger age [11]. $A$ recent study at a tertiary hospital in an Indian subcontinent demonstrated the presence of $B R C A$ pathogenic variant in 13 cases (54\%) among 24 patients who met the criteria for HBOC syndrome, suggestive of the need for a thorough evaluation for hereditary cancer syndromes in these populations [12].

Triple negative breast cancer (TNBC), a subtype of breast cancer, has been reported to have poor outcomes in African American and Latina women. Given its impact on prognosis, the presence of $B R C A$ pathogenic variants in TNBC patients with varied demographic factors was evaluated [13]. In this retrospective study, 450 patients had evaluable genetic test results. Authors reported $139(30.8 \%) B R C A 1(n=106)$ or $B R C A 2(n=32)$ pathogenic variants. In this report, the $B R C A$ pathogenic variant prevalence was: African American (20.4\%), Ashkenazi Jewish (50\%), Asian (28.5\%), Caucasian (33.3\%), and Hispanic (20\%). The prevalence of pathogenic variants also differed by age at diagnosis: < 40 years (43.8\%), 40 49 years $(27.4 \%), 50-59$ years $(25.3 \%), 60-69$ years $(12.5 \%)$, and $>70$ years (16.6\%) [13]. A BRCA1 frameshift pathogenic variant (rs80350973) has also been reported among 125 Chinese patients with TNBC with a prevalence of (7.2\%) [14]. This high prevalence may lend insight to genotype-phenotype correlations for this specific patient population.

Further genetic risk evaluation for $\mathrm{HBOC}$ syndrome should be considered in individuals with a personal history and/or a family history of the following: Breast cancer diagnosed under or equal to age 50 years, TNBC diagnosed under or equal to age 60 years, ovarian cancer, male breast cancer, pancreatic cancer, metastatic breast cancer, metastatic prostate cancer, and multiple $B R C A$-associated cancers in the same person [8,15-17].

\section{Other hereditary breast cancer syndromes among Asians}

Li-Fraumeni syndrome: Pathogenic variants in the TP53 gene are associated with Li-Fraumeni syndrome (LFS), char- acterized by early-onset breast cancer, soft tissue and bone sarcomas, leukemia, broncho-alveolar cancer, adrenocortical cancer, choroid plexus, and brain cancers. The lifetime cancer risks for individuals with LFS are significant. However, cancer-specific risks for individuals with LFS are currently unknown and are likely influenced by genotype, personal risk factors, environment, and modifier genes. The risk of developing at least one LFS-associated cancer is estimated to be $50 \%$ by age 30 and $90 \%$ by age 60 [18].

A recent study reported nine different TP53 pathogenic variants in an attempt to better determine the cancer-specific risks between Korean and Caucasian patients with LFS. Two novel frameshift pathogenic variants were reported in the TP53 gene at p.Pro98Leufs 25 and p.Pro27Leufs ${ }^{*} 17$. Recurrent missense pathogenic variants were also reported at codons 31 (p.Val31l1e), 175 (p.Arg175His) and 273 (p.Arg273Cys) [19].

Individuals with LFS have a high lifetime risk of developing cancer, will often develop cancer at an early age, and may develop more than one primary cancer throughout their lifetime. Although germline TP53 pathogenic variants are rare, comprehensive hereditary cancer risk assessment needs to be performed in any individual diagnosed with breast cancer under age 31 years and in families with multiple, rare tumors occurring at early ages [18].

Cowden syndrome: Pathogenic variants in the PTEN gene are associated with PTEN hamartoma tumor syndrome (PHTS) a spectrum of highly variable conditions with overlapping features including Cowden syndrome (CS) [20]. CS is characterized by hamartomas, papillomas of the lips, mucous membranes, acral skin keratosis, and macrocephaly. Affected individuals are also at an increased risk to develop breast cancer (81-85\%), endometrial cancer (28\%), non-medullary thyroid cancers $(35 \%)$, kidney cancer $(35 \%)$, colon cancer $(9 \%)$, and melanoma (6\%) [21].

Germline PTEN (MMAC1) analysis was performed in 12 Japanese patients with a clinical diagnosis of CS and their relatives [22]. This study reported novel missense and nonsense mutations with their novel techniques. Previous studies also demonstrated germline mutations in PTEN/MMAC1 on chromosome $10 q 23$ in $40-80 \%$ of the patients with CS. It was concluded that further functional and molecular epidemiologic studies need to be performed to confirm phenotype-genotype associations in patients with CS, especially those of minority groups [22].

Revised PHTS clinical diagnostic criteria were proposed after a systematic search and review of the medical literature [23]. Recommendations for germline testing were made whenever possible to confirm a clinical diagnosis in the patient and to facilitate cascade testing in subsequent family members [23]. Based on an individual's presenting clinical manifestations, healthcare professionals may also utilize Cleveland Clinic's online risk assessment tool to estimate a patient's likelihood of harboring a PTEN gene pathogenic variant and recommend for referral to a genetics professional to discuss genetic testing [20]. 
Citation: Kamaraju S, Jacquart A, Stachowiak S, Geurts J, Depas M, et al. (2019) Disparities in Genetic Referrals for Breast Cancer among the Asian Immigrant Populations: How Can We Eliminate Them?. Ann Public Health Reports 3(1):35-40

Lynch syndrome: Pathogenic variants in the mismatch repair (MMR) genes: $M L H 1, M S H 2, M S H 6$ and $P M S 2$ along with deletions of EPCAM are associated with Lynch syndrome (LS) also known as hereditary non-polyposis colorectal cancer (HNPCC). Pathogenic variants in each gene associated with LS have varying levels of cancer risk and can even present differently within families. LS is characterized by an increased risk of developing colorectal, uterine, and ovarian cancer, as well as many other types of cancer including, gastric, small bowel, kidney, central nervous system, biliary tract, pancreatic, prostate and skin [24]. Some studies have suggested an increased risk for breast cancer in LS patients $[25,26]$.

A retrospective study was conducted in Singapore General Hospital in a total of 240 patients under the age of 50 who were diagnosed between January 2006 and December 2010 [27]. In this small retrospective study, authors demonstrated MMR deficiency by immunohistochemistry (IHC) staining in a total of $21 \%(n=51)$ patients. Notably, at the time of this study $86 \%(n=44)$ of patients would have been missed if Amsterdam criteria were solely used for hereditary colon cancer risk evaluation. Authors concluded that mismatch repair deficiency by IHC should be offered to all young ( $\leq$ 50 years) Asians with colorectal cancer. Additionally, a prevalence of $4.19 \%$ for LS has been documented among Asian immigrants with colon cancer [28]. Importantly, this prevalence was much higher (16\%) in patients with a younger age of onset $(<50$ years), and all of the patients studied had a family history of colon or gastric cancer [28].

Germline genetic evaluation for LS should be considered in individuals with a personal history of colorectal or endometrial cancer diagnosed under age 50 years, synchronous or metachronous LS-associated cancer, an individual colorectal or endometrial cancer with a tumor that demonstrates MMR deficiency (either by microsatellite instability testing or loss of MMR protein expression). Genetic risk assessment for LS may also be considered in individuals with a significant family history of LS-associated cancers [24].

Peutz-Jeghers syndrome: Pathogenic variants in the STK11 gene are associated with Peutz-Jeghers syndrome (PJS), characterized by hamartomatous lesions of gastrointestinal tract, mucocutaneous pigmentation and multiple other cancers including, breast (45-50\%), colon (39\%), gastric (29\%), pancreas (11-36\%) small bowel (13\%), lung (15-17\%), endometrium (9\%), ovary and sex cord tumors (18-21\%) [29,30].

To date, few large studies have been conducted to characterize the clinical characteristics and cancer risks associated with PJS in the Asian population. In one study, seven novel and four recurrent STK11 pathogenic variants (c.658C $>C / T$, c.890G > G/A, c.1062 C > C/G, and c. $290+1 G>G / A)$ were identified in Chinese children with PJS. The children developed polyps at an early age (with a median age of onset of 2 years and four months) and in all parts of the gastroenterological tract [31].

PJS should be strongly suspected in individuals with histologically confirmed PJS-type hamartomatous polyps and characteristic mucocutaneous pigmentation. However, it is recommended that affected individuals be referred to a specialty care clinic for comprehensive management of cancer risks due to the complexity of this rare syndrome [30].

Hereditary diffuse gastric cancer syndrome: Pathogenic variants in the $C D H 1$ gene are associated with hereditary diffuse gastric cancer (HDGC) syndrome, characterized by a significant increased risk for diffuse gastric cancer in men (67-80\%) and women (56-83\%) and lobular breast cancer in women (36-52\%) [32]. HDGC should be suspected in families with two or more diagnoses of gastric cancer and one family member with a confirmed diagnosis of diffuse gastric cancer, in a single individual with a confirmed diagnosis of diffuse gastric cancer under age 40 years, or in families with both diffuse gastric cancer and lobular breast cancer with one diagnosis under age 50 years [33].

Although the overall incidence of stomach cancer has declined globally since the 1970s, it remains one of the more common gastrointestinal malignancies observed in populations worldwide. Increased gastric cancer risks are observed in Lynch syndrome, Li-Fraumeni syndrome, PeutzJegher syndrome, and particularly in East Asian families $[34,35]$. Although gastric cancer rates are lower among Asian Americans (Korean, Japanese, Chinese and Filipino Americans) compared to native Asians from these countries [35], the international gastric cancer linkage consortium (IGCLC) consensus guidelines for HDGC selection need to be carefully considered in cultural context during hereditary risk evaluation [34].

For individuals who test positive for apathogenic variant in the $C D H 1$ gene, a prophylactic total gastrectomy is recommended between ages 18 and 40 due to the high mortality rate associated with invasive disease and the low rate of detection by endoscopic screening due to the diffuse gastric pathology [33]. Additionally, woman who carry a pathogenic variant in the $C D H 1$ gene are candidates for high risk breast management and earlier screening including mammogram, breast MRI, and consideration of risk-reducing mastectomy [33]. Therefore, genetic counseling and testing for HDGC are imperative for proper risk assessment for Asian families.

\section{The Barriers}

\section{Patient and provider-related factors and barri- ers that play a crucial role in genetic referrals among the minority communities}

Low rates of genetic testing for women with ovarian cancer among the minority communities as a whole have been reported [36]. In their electronic chart based assessment, the authors reported their primary outcome as the placement of genetic referrals and counseling. A secondary outcome was the completion of genetic testing. Among the 236 eligible subjects, there were significant differences in the placement of genetic referral patterns by race. A total of $61 \%$ of the Caucasians, $40 \%$ of Asians, $38 \%$ Latina and $33 \%$ of Blacks received referrals ( $p-0.035$ ) [36]. Among these study subjects, only $36 \%$ of women diagnosed with ovarian cancer underwent counseling, and 33\% received genetic testing [36]. Authors 
Citation: Kamaraju S, Jacquart A, Stachowiak S, Geurts J, Depas M, et al. (2019) Disparities in Genetic Referrals for Breast Cancer among the Asian Immigrant Populations: How Can We Eliminate Them?. Ann Public Health Reports 3(1):35-40

also reported an association of English language, private insurance, and serous histology as critical factors for the disparities genetic referrals. One study demonstrated disparities in genetic referrals genetic testing among young African American women [37]. The investigators evaluated factors associated with genetic counseling and testing among Black women diagnosed with breast cancer $\leq$ age 50 , recruited through the Florida State Cancer Registry. Among the 440 eligible participants, only 224 (51\%) received genetic counseling and/or $\mathrm{HBOC}$ testing. Other variables such as higher education level, income, the presence of private insurance, triple negative breast cancer, and young age ( $\leq 45$ years) at diagnosis influenced the association with genetic referrals [37]. The authors also reported socioeconomic factors playing a vital role in the uptake of genetic referrals and testing patterns.

\section{Patient-related barriers}

Among US immigrant and refugee populations, low rates of literacy, and high rates of poverty are common in certain groups [38]. These socioeconomic factors contribute to several barriers such as access to transportation, and healthcare insurance, limiting one's own ability to attend to their medical needs in a timely manner $[39,40]$. A cross-sectional study from three different Cancer Control Modules (2005, 2010, 2015), was performed from in-person interview and obtained self-reported data [41]. Eligible participants included women with a history of breast and or ovarian cancer, with an outcome being genetic testing for cancer risk. Of 47,218 women, breast cancer history was reported in $2.7 \%$, of which $35.6 \%$ met one or more criteria for genetic testing, $29 \%$ discussed, and $20.2 \%$ were advised to undergo genetic testing. But, only $15.3 \%$ the women underwent testing [41]. The authors concluded that fewer than one in five individuals with a history of breast or ovarian cancer had undergone genetic testing and the most have never discussed testing with their health care provider [41].

Other important, and yet frequently unnoticed barriers, include interpreter services for non-English speaking Asian patients. While experienced interpreters are available at most healthcare institutions, in-person language services may not be available for every Asian language and connectivity to telephonic services may be poor $[42,43]$. Additionally, even experienced interpreters may have some difficulty translating complex genetic terminology, which may influence a patient's understanding and decision making [44]. There exists a language barrier not only in direct conversation with patients but also in written language [45]. While there are many written resources available to healthcare providers to offer Spanish speaking patients, there are very few in other languages.

\section{Provider-related barriers}

Among non-cancer healthcare providers, physicians' understanding of general cancer risk assessment and identification of high risk families requiring genetic referrals also vary widely [46]. Identification of familial cancer syndromes are somewhat of a challenge to many primary care providers given limited training and exposure to cancer risk assessment, as well as rapid developments in molecular diagnostics $[47,48]$. This was illustrated by a successful web based curriculum that led to an increase in the confidence levels and referral behaviors of primary care providers engaged in genetic counseling [48]. Investigators randomized 121 primary care providers (PCPs) to an intervention of web-based curriculum focusing on communication skills and basics of genetic screening and knowledge against a control group, where PCPs were provided with standard education materials. The intervention group demonstrated significant learning, knowledge, retention, and shared decision practices with the web-based learning. This highlights the emphasis on the utilization of web-based study materials and a system based approach to enhance providers' learning in their busy day to day schedules [48].

\section{Conclusions and Future Directions}

On the ethical principles of justice and fairness, Asian immigrant and refugee populations may not be equally served in healthcare if physicians and healthcare providers practice solely within the Western medical system ideology. Hospital institutions should advocate for cultural competency training programs for their physicians and advanced care practitioners. This specialized training will focus on better care for our minority groups affected by ethnic and racial disparities in genomic healthcare [44].

Given the increasing trends of cancer incidence among the migrant populations and the potential for some differences in the prevalence of some mutations, emphasis should be given to promote the awareness of familial cancer syndromes. Integration of novel electronic platforms for providers and patients will enhance even distance/online \& web-based learning easily accessible. Additional efforts to support young individuals of Asian immigrant and refugee populations to become interested in pursuing a career in genomic healthcare to promote a new generation of bilingual and bicultural advanced care practitioners.

\section{References}

1. Kwong A, Chu AT, Wu CT, et al. (2014) Attitudes and compliance of clinical management after genetic testing for hereditary breast and ovarian cancer among high-risk Southern Chinese females with breast cancer history. Fam Cancer 13: 423-430.

2. Rolnick SJ, Rahm AK, Jackson JM, et al. (2011) Barriers in identification and referral to genetic counseling for familial cancer risk: The perspective of genetic service providers. J Genet Couns 20: 314-322.

3. Rastogi T, Devesa S, Mangtani P, et al. (2008) Cancer incidence rates among South Asians in four geographic regions: India, Singapore, UK and US. Int J Epidemiol 37: 147-160.

4. Deapen D, Liu L, Perkins C, et al. (2002) Rapidly rising breast cancer incidence rates among Asian-American women. Int J Cancer 99: 747-750.

5. Allford A, Qureshi N, Barwell J, et al. (2014) What hinders minority ethnic access to cancer genetics services and what may help? Eur J Hum Genet 22: 866-874.

6. Featherstone C, Colley A, Tucker K, et al. (2007) Estimating the referral rate for cancer genetic assessment from a systematic review of the evidence. Br J Cancer 96: 391-398. 
Citation: Kamaraju S, Jacquart A, Stachowiak S, Geurts J, Depas M, et al. (2019) Disparities in Genetic Referrals for Breast Cancer among the Asian Immigrant Populations: How Can We Eliminate Them?. Ann Public Health Reports 3(1):35-40

7. Kim H, Choi DH (2013) Distribution of BRCA1 and BRCA2 mutations in Asian patients with breast cancer. J Breast Cancer 16: 357-365.

8. Petrucelli N, Daly MB, Pal T (1993) BRCA1- and BRCA2-Associated hereditary breast and ovarian cancer. GeneReviews, University of Washington, Seattle.

9. Mavaddat N, Peock S, Frost D, et al. (2013) Cancer risks for BRCA1 and BRCA2 mutation carriers: Results from prospective analysis of EMBRACE. J Natl Cancer Inst 105: 812-822.

10. Chen S, Iversen ES, Friebel T, et al. (2006) Characterization of BRCA1 and BRCA2 mutations in a large United States sample. J Clin Oncol 24: 863-871.

11. Wu X, Wu L, Kong B, et al. (2017) The first nationwide multicenter prevalence study of germline BRCA1 and BRCA2 mutations in Chinese ovarian cancer patients. Int J Gynecol Cancer 27: 16501657.

12. Darooei M, Poornima S, Salma BU, et al. (2017) Pedigree and BRCA gene analysis in breast cancer patients to identify hereditary breast and ovarian cancer syndrome to prevent morbidity and mortality of disease in Indian population. Tumour Biol 39.

13. Greenup R, Buchanan A, Lorizio W, et al. (2013) Prevalence of BRCA mutations among women with triple-negative breast cancer (TNBC) in a genetic counseling cohort. Ann Surg Oncol 20: 3254-3258.

14. Liu X, Li H, Shao B, et al. (2017) Identification of recurrent BRCA1 mutation and its clinical relevance in Chinese triple-negative breast cancer cohort. Cancer Med 6: 547-554.

15. Giri VN, Obeid E, Gross L, et al. (2017) Inherited mutations in men undergoing multigene panel testing for prostate cancer: Emerging implications for personalized prostate cancer genetic evaluation. JCO Precision Oncology 1: 1-17.

16. Lowery MA, Wong W, Jordan EJ, et al. (2018) Prospective evaluation of germline alterations in patients with exocrine pancreatic neoplasms. J Natl Cancer Inst 110: 1067-1074.

17. Robson M, Im SA, Senkus E, et al. (2017) Olaparib for metastatic breast cancer in patients with a germline BRCA mutation. $\mathrm{N}$ Engl J Med 377: 523-533.

18. Schneider K, Zelley K, Nichols KE, et al. (1993) Li-Fraumen syndrome. GeneReviews, University of Washington, Seattle.

19. Park KJ, Choi HJ, Suh SP, et al. (2016) Germline TP53 mutation and clinical characteristics of Korean patients with Li-Fraumen syndrome. Ann Lab Med 36: 463-468.

20. Eng C (1993) PTEN Hamartoma tumor syndrome. GeneReviews, University of Washington, Seattle.

21. Tan MH, Mester JL, Ngeow J, et al. (2012) Lifetime cancer risks in individuals with germline PTEN mutations. Clin Cancer Res 18: 400-407.

22. Sawada T, Hamano N, Satoh H, et al. (2000) Mutation analysis of the PTEN/MMAC1 gene in Japanese patients with Cowden disease. Jpn J Cancer Res 91: 700-705.

23. Pilarski R, Burt R, Kohlman W, et al. (2013) Cowden syndrome and the PTEN hamartoma tumor syndrome: Systematic review and revised diagnostic criteria. J Natl Cancer Inst 105: 1607-1616.

24. Kohlmann W, Gruber SB (1993) Lynch syndrome. GeneReviews, University of Washington, Seattle.
25. Espenschied CR, LaDuca H, Li S, et al. (2017) Multigene panel testing provides a new perspective on lynch syndrome. J Clin Oncol 35: 2568-2575.

26. Roberts ME, Jackson SA, Susswein LR, et al. (2018) MSH6 and PMS2 germ-line pathogenic variants implicated in lynch syndrome are associated with breast cancer. Genet Med 20: 1167-1174.

27. Chew MH, Koh PK, Tan M, et al. (2013) Mismatch repair deficiency screening via immunohistochemical staining in young Asians with colorectal cancers. World J Surg 37: 2468-2475.

28. Lee J, Xiao YY, Sun YY, et al. (2017) Prevalence and characteristics of hereditary non-polyposis colorectal cancer (HNPCC) syndrome in immigrant Asian colorectal cancer patients. BMC Cancer 17: 843.

29. Hearle N, Schumacher V, Menko FH, et al. (2006) Frequency and spectrum of cancers in the Peutz-Jeghers syndrome. Clin Cancer Res 12: 3209-3215.

30. McGarrity TJ, Amos Cl, Baker MJ (1993) Peutz-Jeghers syndrome. GeneReviews, University of Washington, Seattle.

31. Huang Z, Miao S, Wang L, et al. (2015) Clinical characteristics and STK11 gene mutations in Chinese children with Peutz-Jeghers syndrome. BMC Gastroenterol 15: 166.

32. Pharoah PD, Guilford P, Caldas C, et al. (2001) Incidence of gastric cancer and breast cancer in $\mathrm{CDH} 1$ (E-cadherin) mutation carriers from hereditary diffuse gastric cancer families. Gastroenterology 121: 1348-1353.

33. Van der Post RS, Vogelaar IP, Carneiro F, et al. (2015) Hereditary diffuse gastric cancer: Updated clinical guidelines with an emphasis on germline $\mathrm{CDH} 1$ mutation carriers. J Med Genet 52: 361-374.

34. Kaurah P, MacMillan A, Boyd N, et al. (2007) Founder and recurrent $\mathrm{CDH} 1$ mutations in families with hereditary diffuse gastric cancer. JAMA 297: 2360-2372.

35. Kim Y, Park J, Nam BH, et al. (2015) Stomach cancer incidence rates among Americans, Asian Americans and Native Asians from 1988 to 2011. Epidemiol Health 37: e2015006.

36. Manrriquez E, Chapman JS, Mak J, et al. (2018) Disparities in genetics assessment for women with ovarian cancer: Can we do better? Gynecol Oncol 149: 84-88.

37. Cragun D, Bonner D, Kim J, et al. (2015) Factors associated with genetic counseling and BRCA testing in a population-based sample of young black women with breast cancer. Breast Cancer Res Treat 151: 169-176.

38. Sentell T, Braun KL (2012) Low health literacy, limited English proficiency, and health status in Asians, Latinos, and other racial/ethnic groups in California. J Health Commun 17: 82-99.

39. Kamaraju S, DeNomie M, Visotcky A, et al. (2018) Increasing mammography uptake through academic-community partnerships targeting immigrant and refugee communities in Milwaukee. WMJ 117: 55-61.

40. Yao N, Hillemeier MM (2014) Disparities in mammography rate among immigrant and native-born women in the U.S.: Progress and challenges. J Immigr Minor Health 16: 613-621.

41. Childers KK, Maggard-Gibbons M, Macinko J, et al. (2018) National distribution of cancer genetic testing in the United States: Evidence for a gender disparity in hereditary breast and ovarian cancer. JAMA Oncol 4: 876-879. 
Citation: Kamaraju S, Jacquart A, Stachowiak S, Geurts J, Depas M, et al. (2019) Disparities in Genetic Referrals for Breast Cancer among the Asian Immigrant Populations: How Can We Eliminate Them?. Ann Public Health Reports 3(1):35-40

42. Hadziabdic E, Hjelm K (2013) Working with interpreters: Practical advice for use of an interpreter in healthcare. Int J Evid Based Healthc 11: 69-76.

43. Hadziabdic E, Heikkila K, Albin B, et al. (2011) Problems and consequences in the use of professional interpreters: Qualitative analysis of incidents from primary healthcare. Nurs Inq 18: 253-261.

44. LeRoy B, Veach PM, Bartels DM (2010) Genetic counseling practice: Advanced concepts and skills. Wiley-Blackwell, Hoboken, NJ.

45. Weil J (2000) Psychosocial genetic counseling. Oxford University Press, Oxford, New York.
46. Vig HS, Armstrong J, Egleston BL, et al. (2009) Cancer genetic risk assessment and referral patterns in primary care. Genet Test Mol Biomarkers 13: 735-741.

47. Bell J (2004) Predicting disease using genomics. Nature 429: 453456.

48. Wilkes MS, Day FC, Fancher TL, et al. (2017) Increasing confidence and changing behaviors in primary care providers engaged in genetic counselling. BMC Med Educ 17: 163.

DOI: $10.36959 / 856 / 489$

Copyright: (c) 2019 Kamaraju S, et al. This is an open-access article distributed under the terms of the Creative Commons Attribution License, which permits unrestricted use, distribution, and reproduction in any medium, provided the original author and source are credited. 